\title{
Serological Response of Cats to Experimental Besnoitia darlingi and Besnoitia neotomofelis Infections and Prevalence of Antibodies to These Parasites in Cats from Virginia and Pennsylvania
}

\author{
Author(s): Alice E. Houk, Alexa C. Rosypal, David C. Grant, J. P. Dubey, Anne M. Zajac, Michael J. \\ Yabsley, and David S. Lindsay \\ Source: Journal of Parasitology, 97(2):259-261. 2011. \\ Published By: American Society of Parasitologists \\ DOI: http://dx.doi.org/10.1645/GE-2626.1 \\ URL: http://www.bioone.org/doi/full/10.1645/GE-2626.1
}

BioOne (www.bioone.org) is a nonprofit, online aggregation of core research in the biological, ecological, and environmental sciences. BioOne provides a sustainable online platform for over 170 journals and books published by nonprofit societies, associations, museums, institutions, and presses.

Your use of this PDF, the BioOne Web site, and all posted and associated content indicates your acceptance of BioOne's Terms of Use, available at www.bioone.org/page/terms of use.

Usage of BioOne content is strictly limited to personal, educational, and non-commercial use. Commercial inquiries or rights and permissions requests should be directed to the individual publisher as copyright holder. 
American Society of Parasitology. Alice E. Houk, Alexa C. Rosypal, David C. Grant, J. P. Dubey, Anne M. Zajac, Michael J. Yabsley, and David S. Lindsay (2011). "Serological Response of Cats to Experimental Besnoitia darlingi and Besnoitia neotomofelis Infections and Prevalence of Antibodies to These Parasites in Cats from Virginia and Pennsylvania," Journal of Parasitology, Vol. 97, No. 2, pp. 259-261. doi: http://dx.doi.org/10.1645/GE-2626.1

\title{
SEROLOGICAL RESPONSE OF CATS TO EXPERIMENTAL BESNOITIA DARLINGI AND BESNOITIA NEOTOMOFELIS INFECTIONS AND PREVALENCE OF ANTIBODIES TO THESE PARASITES IN CATS FROM VIRGINIA AND PENNSYLVANIA
}

\author{
Alice E. Houk, Alexa C. Rosypal ${ }^{\star}$, David C. Grant $†$, J. P. Dubey $\ddagger$, Anne M. Zajac, Michael J. Yabsley $\S$, and \\ David S. Lindsay\| \\ Department of Biomedical Sciences and Pathobiology, Virginia-Maryland Regional College of Veterinary Medicine, Virginia Tech, Blacksburg, \\ Virginia 24061-0342. e-mail: lindsayd@vt.edu
}

\begin{abstract}
Besnoitia darlingi and Besnoitia neotomofelis are cyst-forming tissue apicomplexan parasites that use domestic cats (Felis domesticus) as definitive hosts and opossums (Didelphis virginiana) and Southern Plains woodrats (Neotoma micropus) as intermediate hosts, respectively. Nothing is known about the prevalence of B. darlingi or B. neotomofelis in cats from the United States. Besnoitia darlingi infections have been reported in naturally infected opossums from many states in the United States, and B. neotomofelis infections have been reported from Southern Plains woodrats from Texas, but naturally infected cats have not been identified. The present study examined the $\mathrm{IgG}$ antibody response of cats to experimental infection (B. darlingi $\mathrm{n}=1$ cat; B. neotomofelis $\mathrm{n}=3$ cats). Samples from these cats were used to develop an indirect immunofluorescent antibody test (IFAT), which was then used to examine seroprevalence of $\mathrm{IgG}$ antibodies to tachyzoites of $B$. darlingi and B. neotomofelis in a population of domestic cats from Virginia $(\mathrm{N}=$ 232 cats) and Pennsylvania $(\mathrm{N}=209)$. The serum from cats inoculated with $B$. darlingi or $B$. neotomofelis cross-reacted with each other's tachyzoites. The titers to heterologous tachyzoites were 1 to 3 dilutions lower than to homologous tachyzoites. Sera from $B$. darlingi- or B. neotomofelis-infected cats did not react with tachyzoites of Toxoplasma gondii or Neospora caninum or merozoites of Sarcocystis neurona using the IFAT. Antibodies to B. darlingi were found in 14\% and $2 \%$ of cats from Virginia and Pennsylvania, respectively. Antibodies to B. neotomofelis were found in 5\% and $4 \%$ of cats from Virginia and Pennsylvania, respectively. Nine cats from Virginia and 1 cat from Pennsylvania were positive for both.
\end{abstract}

Besnoitia is a group of cyst-forming tissue apicomplexan parasites, some of which are of veterinary significance. Several Besnoitia species cause fatal infections and economic losses in domestic animals and wildlife in various countries (Dubey, 2009; Mehlhorn et al., 2009). Besnoitia darlingi uses cats and opossums in its life cycle. The Virginia opossum (Didelphis virginiana) is the intermediate host, and domestic cats (Felis domesticus) are definitive hosts. Cats become infected by ingesting tissue cysts from opossums and produce oocysts that are shed in the feces (Smith and Frenkel, 1977, 1984; Dubey et al., 2002). Ten species of Besnoitia have been described, and domestic cats are the definitive hosts for the 4 species with known life cycles (reviewed by Dubey and Yabsley, 2010; Kiehl et al., 2010). The life cycles of the remaining 6 Besnoitia species are presently unknown.

Little is known about the epidemiology of $B$. darlingi in cats from the United States. Besnoitia darlingi tissue cysts or antibodies have been described in opossums from Illinois, Indiana, Kentucky, Louisiana, Michigan, Mississippi, Missouri, and Texas (reviewed by Houk et al., 2010). Natural B. darlingi infections have been diagnosed in United States opossums (Houk et al., 2010); however, naturally infected cats have not yet been

Received 11 August 2010; revised 20 October 2010; accepted 21 October 2010.

* Department of Natural Sciences and Mathematics, College of Science, Technology, Engineering, and Mathematics, Johnson C. Smith University, Charlotte, North Carolina 28216.

$\dagger$ Department of Small Animal Clinical Sciences, Virginia-Maryland Regional College of Veterinary Medicine, Virginia Tech, Blacksburg, Virginia 24061-0342.

$\$$ Animal Parasitic Diseases Laboratory, Agricultural Research Service, United States Department of Agriculture, Animal and Natural Resources Institute, Beltsville Agricultural Research Center, BARCEast, Building 1001, Beltsville, Maryland 20705-2350.

$\S$ Warnell School of Forestry and Natural Resources and the Southeastern Cooperative Wildlife Disease Study, Department of Population Health, College of Veterinary Medicine, University of Georgia, Athens, Georgia 30602 .

" To whom correspondence should be addressed.

DOI: 10.1645/GE-2626.1 identified (Dubey et al., 2002), and there is no information regarding the prevalence of $B$. darling $i$ in cats in the United States. This is due to the difficulty in identifying Besnoitia spp. oocysts and in differentiating them from oocysts of Toxoplasma gondii in cat feces. Moreover, Besnoitia sp. antigen is unavailable commercially and must be grown in cell culture systems.

Besnoitia neotomofelis Dubey and Yabsley, 2010 was described from naturally infected Southern Plains woodrats (Neotoma micropus). Laboratory mice (Mus musculus) and rats (Rattus norvegicus) have been shown to be intermediate hosts, and cats have been identified as the definitive host (Dubey and Yabsley, 2010). Phylogenetic analysis using the $18 \mathrm{~S}$ rRNA gene and the ITS-1 region indicated that $B$. neotomofelis is distinct from $B$. darlingi, Besnoitia wallacei, and Besnoitia oryctofelisi, all of which utilize cats as a definitive host (Dubey and Yabsley, 2010).

The present study was conducted to investigate the serological response of cats to inoculation with tachyzoites of $B$. darling $i$ and $B$. neotomofelis and to determine the seroprevalence of antibodies to these 2 Besnoitia species in cats from Virginia and Pennsylvania. In addition, we also evaluated the indirect immunofluorescent antibody test (IFAT) using sera from experimentally infected cats for cross-reactivity to stages of $T$. gondii, Neospora caninum, and Sarcocystis neurona.

\section{MATERIALS AND METHODS}

\section{Parasite cultivation}

Tachyzoites of $B$. darlingi (strain of Dubey et al., 2002) and $B$. neotomofelis (strain of Dubey and Yabsley, 2010) were grown separately in human foreskin fibroblasts (Hs68, ATCC CRL1635, Manassas, Virginia) that had grown to confluence in $75-\mathrm{mm}^{2}$ tissue culture flasks. Growth media consisted of 10\% Fetal Bovine Serum (FBS) (v/v) in RPMI 1640 medium, supplemented with $100 \mathrm{U}$ penicillin/ml and $100 \mathrm{mg}$ streptomycin/ $\mathrm{ml}$. After monolayers had reached confluence, the growth medium was removed and replaced with a maintenance medium that was identical to the former, except the volume of FBS was $2 \%(\mathrm{v} / \mathrm{v})$. Flasks were incubated at $37 \mathrm{C}$ in a humidified incubator containing $5 \% \mathrm{CO}_{2}$ and $95 \%$ air. Additionally, tachyzoites of the RH strain (Sabin, 1941) of T. gondii and the NC-1 strain (Dubey et al., 1988) of N. caninum were grown in Hs68 
cells, and merozoites of the SN-37R isolate (Sofaly et al., 2002) of $S$ neurona were grown and maintained in African green monkey (Cercopithecus aethiops) kidney cells (CV-1, ATTC CCL-70, ATCC) using techniques identical to those described for Hs68 cells.

\section{Experimental inoculation of cats}

Four 3- to 4-mo-old cats from the parasite-free colony (Dubey, 1995) housed at the United States Department of Agriculture, Animal Parasitic Diseases Laboratory, Beltsville, Maryland, were used to examine the $\operatorname{IgG}$ antibody response to $B$. darlingi and $B$. neotomofelis. Cat 77 was inoculated subcutaneously with $1 \times 10^{6}$ tachyzoites of $B$. darlingi, and serum samples were collected on days 0,7 , and 36 postinoculation (PI). Two cats $(90,91)$ were fed infected mouse tissues containing $B$. neotomofelis tissue cysts; the mice had been fed B. neotomofelis oocysts 5 mo earlier. Both cats remained asymptomatic and shed $B$. neotomofelis oocysts 12-25 (cat 90) and 13-20 (cat 91) day PI. Sera samples were collected from these cats on days 0, 26, and 40 PI. An additional sample from cat 90 was collected on day 10 PI. Cat 78 was inoculated subcutaneously with $1 \times 10^{6}$ cell culture-derived $B$. neotomofelis tachyzoites, and serum samples were collected on days 0,7 , and 36 PI.

Sera samples were shipped by overnight carrier to the Center for Molecular Medicine and Infectious Diseases (CMMID), Virginia-Maryland Regional College of Veterinary Medicine, Virginia Tech, Blacksburg, Virginia, and stored at $4 \mathrm{C}$ until examined. Sera samples were used at an initial dilution of 1:50 and end-point titrated (Table I). Sera samples from all 4 cats were examined for titers against $B$. darlingi and B. neotomofelis on each sample collection day. Sera samples collected from last evaluation period (days 36 or $40 \mathrm{PI}$ ) were used to examine specificity of the test using T. gondii, $N$. caninum, and $S$. neurona stages as antigens.

\section{Feline samples and immunofluorescent antibody test}

Feline serum or plasma samples were obtained from 441 cats for the present study (Hsu et al., 2010). Serum or plasma samples were collected from 232 cats from Virginia as part of an ongoing study of kidney disease conducted at the Department of Small Animal Clinical Science, at the Virginia-Maryland Regional College of Veterinary Medicine, Blacksburg, Virginia. An additional 209 serum samples were acquired from an animal shelter in Philadelphia, Pennsylvania. These samples were initially used in a study of the seroprevalence of $T$. gondii (Dubey et al., 2008). Serum and plasma samples were stored at $-20 \mathrm{C}$ until used.

For collection of parasites for the IFAT, infected Hs68 or CV-1 cells were removed with a cell scraper, the media mixture was filtered through 3- $\mu \mathrm{m}$ polycarbonate filters (GE Water and Process Technologies, Minnetonka, Minnesota), and parasites were pelleted by centrifugation. After 3 washes in phosphate-buffered saline (PBS), the cell suspension containing approximately $0.5-1 \times 10^{4}$ tachyzoites/merozoites in $25 \mu \mathrm{l}$ of PBS was dispensed into each well of a 12-well Teflon-coated IFAT slide (Fisher Scientific, Pittsburgh, Pennsylvania). Antigen-containing slides were then left to dry at room temperature for 4 to $12 \mathrm{hr}$ and stored at $-20 \mathrm{C}$ until used.

Cat serum or plasma samples were diluted at 1:100 in PBS, and $25 \mu$ were pipetted into each well of the antigen-containing slides. The slides were incubated for $30 \mathrm{~min}$ at room temperature in a humidified chamber. Subsequent to 2 consecutive washes in PBS, fluorescence-labeled goat anti-rabbit IgG (Kirkegaard and Perry Labs Inc., Gaithersburg, Maryland) was diluted 1:5 in PBS, and $25 \mu 1$ aliquots were added to each well of the slides. The slides were incubated for $30 \mathrm{~min}$ at room temperature in a humidified chamber. Following 3 consecutive washes in PBS, slides were mounted in Fluoromount-G (Southern Biotechnology Associates Inc., Birmingham, Alabama), and assessed using an Olympus BX60 epifluorescent microscope equipped with differential contrast optics (Olympus America Inc., Center Valley, Pennsylvania). Samples that exhibited fluorescence of the entire parasitic surface were considered to be positive.

\section{RESULTS}

None of the experimentally infected cats was seropositive to $B$. darlingi or B. neotomofelis prior to inoculation (Table I). All cats seroconverted to the Besnoitia species inoculated and demon-
TABLE I. Serological response (reciprocal antibody titer) of cats inoculated separately with $1 \times 10^{6}$ tachyzoites of Besnoitia darlingi or B. neotomofelis and tissue cysts of $B$. neotomofelis and examined using the indirect fluorescent antibody test at various days postinoculation (PI).

\begin{tabular}{llccc}
\hline Cat & Inoculum & Day PI & $\begin{array}{c}\text { B. darlingi } \\
\text { titer }\end{array}$ & $\begin{array}{c}\text { B. neotomofelis } \\
\text { titer }\end{array}$ \\
\hline $77^{*}$ & B. darlingi & 0 & $<1: 50$ & $<1: 50$ \\
77 & B. darlingi & 7 & $1: 800$ & $1: 200$ \\
77 & B. darlingi & 36 & $1: 800$ & $1: 200$ \\
$78^{*}$ & B. neotomofelis & 0 & $<1: 50$ & $<1: 50$ \\
78 & B. neotomofelis & 7 & $<1: 50$ & $<1: 50$ \\
78 & B. neotomofelis & 36 & $1: 200$ & $1: 200$ \\
$90 \dagger$ & B. neotomofelis & 0 & $<1: 50$ & $<1: 50$ \\
90 & B. neotomofelis & 10 & $<1: 50$ & $<1: 50$ \\
90 & B. neotomofelis & 26 & $1: 200$ & $1: 200$ \\
90 & B. neotomofelis & 40 & $1: 200$ & $1: 800$ \\
$91 \dagger$ & B. neotomofelis & 0 & $<1: 50$ & $<1: 50$ \\
91 & B. neotomofelis & 26 & $<1: 50$ & $<1: 50$ \\
91 & B. neotomofelis & 40 & $1: 100$ & $1: 200$ \\
\hline
\end{tabular}

* Tachyzoites

+ Tissue cysts.

strated cross-reactive reactions with the Besnoitia species not inoculated. None of the serum samples from cats experimentally inoculated with $B$. darling $i$ or $B$. neotomofelis collected on any examination day PI reacted with stages of $T$. gondii, $N$. caninum, or $S$. neurona in the IFAT. No reactivity to $B$. darling $i$ was observed in cats that had previously tested positive for antibodies to T. gondii or S. neurona (Dubey et al., 2008; Hsu et al., 2010).

Antibodies to $B$. darling $i$ and $B$. neotomofelis were found in $14 \%$ and $5 \%$ cats, respectively, from Virginia. Nine of these cats were positive for both $B$. darlingi and $B$. neotomofelis. Antibodies to $B$. darlingi and B. neotomofelis were found in 3 and 5 cats, respectively, from Pennsylvania. One of these cats was positive for both $B$. darlingi and $B$. neotomofelis.

\section{DISCUSSION}

Besnoitia spp. parasitize domestic and wild animals. In some parts of the world, these parasites cause serious economic losses in cattle. Ten species of Besnoitia have presently been named (Dubey and Yabsley, 2010), but the life cycle is not known for any species. Cats have been identified as the definitive host for $B$. wallacei, $B$. darling, $B$. oryctofelisi, and the recently recognized species $B$. neotomofelis (Dubey and Yabsley, 2010).

Smith and Frenkel (1984) examined the serological response to $B$. darlingi inoculation of opossums and cats using tachyzoites of Besnoitia jellisoni as an antigen in the Sabin-Feldmen dye test (SFDT). Sera samples from 5 opossums inoculated with tissue cysts oocysts, tachyzoites, or a mixture of all 3 stages seroconverted to $B$. jellisoni in the SFDT. They (Smith and Frenkel, 1984) reported that 2 cats given $B$. darlingi oocysts did not seroconvert in the SFDT or excrete oocysts. Five of 14 cats given $B$. darling $i$ tissue cysts demonstrated seroconversion to $B$. jellisoni in the SFDT, and all 14 cats excreted oocysts (Smith and Frenkel, 1984). This suggests that intestinal infection with stages of $B$. darlingi does not induce a strong serological response. Interestingly, 2 of 4 cats challenged with $B$. darling $i$ after excreting oocysts were susceptible to repeat oocyst excretion (Smith and Frenkel, 1984). The cat in the present study inoculated with 
tachyzoites seroconverted to homologous antigen in the IFAT. The results of our study are difficult to compare to those of Smith and Frenkel (1984) because different serological tests and different strains of $B$. darling $i$ and modes/stages were used to administer parasites. We demonstrated cross-reactivity of cat $B$. darlingi anti-sera with $B$. neotomofelis antigen and cross-reactivity of cat $B$. neotomofelis anti-sera with $B$. darlingi in the present study, and this is consistent with the finding of cross-reactivity of cat $B$. darlingi anti-sera with $B$. jellisoni antigen reported by Smith and Frenkel (1984). We also demonstrate that antibodies in cat sera to $B$. darlingi or $B$. neotomofelis were not cross-reactive to $T$. gondii, $N$. caninum, or $S$. neurona in the IFAT. Lunde and Jacobs (1965) demonstrated cross-reactivity of B. jellisoni and T. gondii in rabbit sera using the hemagglutination test, but not the dye test.

This is the first report to use the IFAT to examine the prevalence of exposure of domestic cats to $B$. darlingi. In the United States, $B$. darlingi tissue cysts have been reported from 1 of 1 opossums from Illinois (Flatt et al., 1971), 6 of 6 from Indiana (Jack et al., 1989), 1 of 5 (20\%) from Kentucky (Conti-Diaz et al., 1970), 1 of 1 from Louisiana (Shaw et al., 2009), 15 of 137 (11\%) from Michigan (Elsheikha et al., 2003), 2 of 2 from Mississippi (Dubey et al., 2002), 7 of 12 (58\%) from Missouri (Flatt et al., 1971; Smith and Frenkel, 1977), and 1 of 1 Texas (Stabler and Welch, 1961). Antibodies to B. darling $i$ have been described in 14 of $30(47 \%)$ opossums from southern Louisiana using the IFAT (Houk et al., 2010). It is likely that $B$. darlingi is present throughout the range of opossums in North America.

Dubey and Yabsley (2010) reported that acid-pepsin-digested tissues from 1 of 38 woodrats from Texas were positive for $B$. neotomofelis. Tissue cysts of $B$. neotomofelis collected from experimentally infected rodents were infectious for 7 of 13 cats, which excreted few oocysts in their feces (Dubey and Yabsley, 2010). Extraintestinal stages of $B$. neotomofelis have been demonstrated in experimentally infected cats. No other studies have been conducted on the prevalence of $B$. neotomofelis in woodrats or other potential intermediate hosts.

\section{LITERATURE CITED}

Conti-Diaz, I. A., C. Turner, D. T. Tweeddale, and M. L. Furcolow. 1970. Besnoitiasis in the opossum (Didelphis marsupialis). Journal of Parasitology 56: 457-460.

Dubey, J. P. 1995. Duration of immunity to shedding of Toxoplasma gondii oocysts by cats. Journal of Parasitology 81: 410-415. . 2009. The evolution of the knowledge of cat and dog coccidia. Parasitology 136: 1469-1475.

, C. R. Bhatia, M. R. Lappin, L. R. Ferreira, A. Thorn, and O. C. H. Kwok. 2008. Seroprevalence of Toxoplasma gondii and Bartonella spp. antibodies in cats from Pennsylvania. Journal of Parasitology 95: 578-580.

, A. L. Hattel, D. S. Lindsay, and M. J. Topper. 1988. Neonatal Neospora caninum infection in dogs: Isolation of the causative agent and experimental transmission. Journal of the American Veterinary Medical Association 193: 1259-1263.
- D. S. Lindsay, B. M. Rosenthal, C. Sreekumar, D. E. Hill, S. K. Shen, O. C. H. Kwok, L. G. Rickard, S. S. Black, and A. RASHMIR-RAVENC. 2002. Establishment of Besnoitia darlingi from opossums (Didelphis virginiana) in experimental intermediate and definitive hosts, propagation in cell culture, and description of ultrastructural and genetic characteristics. International Journal for Parasitology 32: 1053-1064.

— AND M. J. Yabsley. 2010. Besnoitia neotomofelis n. sp. (Protozoa: Apicomplexa) from the Southern Plains wood rat (Neotoma micropus). Parasitology 21: 1-17.

Elsheikha, H. M., L. S. Mansfield, S. D. Fitzgerald, and M. A. Saeed. 2003. Prevalence and tissue distribution of Besnoitia darlingi cysts in the Virginia opossum (Didelphis virginiana) in Michigan. Veterinary Parasitology 115: 321-327.

Flatt, R. E., L. R. Nelson, and N. M. Patton. 1971. Besnoitia darlingi in the opossum (Didelphis marsupialis). Laboratory Animal Science 21: 106-109.

Houk, A. E., D. G. Goodwin, A. M. Zajac, S. C. Barr, J. P. Dubey, And D. S. Lindsay. 2010. Prevalence of antibodies to Trypanosoma cruzi, Toxoplasma gondii, Encephalitozoon cuniculi, Sarcocystis neurona, Besnoitia darlingi, and Neospora caninum in North American opossums, Didelphis virginiana, from southern Louisiana. Journal of Parasitology 96: 1119-1122.

Hsu, V., D. G. Grant, J. P. Dubey, A. M. Zajac, And D. S. LindSAY. 2010. Prevalence of antibodies to Sarcocystis neurona in cats from Virginia and Pennsylvania. Journal of Parasitology 96: 800801.

Jack, S. W., W. G. van Alstine, And J. Swackhamer. 1989. Besnoitiasis in Indiana opossums. Journal of Veterinary Diagnostic Investigation 1: 189-191.

Kiehl, E., A. O. Heydorn, E. Schein, K. A. Al-Rasheid, J. Selmair, F. Abdel-Ghaffar, and H. Mehlhorn. 2010. Molecular biological comparison of different Besnoitia species and stages from different countries. Parasitology Research 106: 889-894.

Lunde, M. N., AND L. JACOBs.1965. Antigenic relationship of Toxoplasma gondii and Besnoitia jellisoni. Journal of Parasitology 51: 273-276.

Mehlhorn, H., S. Klimpel, E. Schein, A. O. Heydorn, S. Al-Quraishy, And J. Selmair. 2009. Another African disease in Central Europe: Besnoitiosis of cattle. I. Light and electron microscopical study. Parasitology Research 104: 861-868.

SABIN, A. B. 1941. Toxoplasmic encephalitis in children. Journal of the American Medical Association 116: 801-807.

Shaw, S., B. Grasperge, J. Nevarez, S. Reed, L. Long, N. Rademacher, and D. Sánchez-Migallón Guzmán. 2009. Besnoitia darlingi infection in a Virginia opossum (Didelphis virginiana). Journal of Zoo and Wildlife Medicine 40: 220-223.

Smith, D. D., And J. K. Frenkel. 1977. Besnoitia darlingi (Protozoa: Toxoplasmatinae): Cyclic transmission by cats. Journal of Parasitology 63: 1066-1071.

$\longrightarrow$, AND - 1984. Besnoitia darlingi (Apicomplexa, Sarcocystidae, Toxoplasmatinae): transmission between opossums and cats. Journal of Protozoology 31: 584-587.

Sofaly, C. D., S. M. Reed, J. C. Gordon, J. P. Dubey, M. L. Ogleebee, C. J. Nuoku, D. L. Grover, and W. J. Saville. 2002. Experimental induction of equine protozoan myeloencephalitis (EPM) in the horse: Effect of Sarcocystis neurona sporocyst inoculation dose on the development of clinical neurologic disease. Journal of Parasitology 88: $1164-1170$.

Stabler, R. M., and K. Welch. 1961. Besnoitia from an opossum. Journal of Parasitology 47: 576. 